\title{
A MODEL FOR THE DYNAMICAL PROCESS OF CLUSTER FORMATION.
}

\author{
Dirk Aeyels* Filip De Smet*
}

\author{
* SYSTeMS Research Group, \\ Ghent University, \\ Technologiepark-Zwijnaarde 914, \\ 9052 Zwijnaarde, Belgium
}

\begin{abstract}
The formation of several clusters, arising from attracting forces between non-identical entities or agents, is a phenomenon observed in diverse fields. Think of people gathered through a mutual interest, pricing policy of different distributors in an economic market or clustering of oscillators in brain cells. We introduce a dynamical model of mutually attracting agents for which we prove that the long term behavior consists of agents organized into several groups or clusters. We have completely characterized the cluster structure by means of a set of inequalities in the parameters of the model and have identified the intensity of the attraction as a key parameter governing the transition between different cluster structures. We illustrate the relation with the Kuramoto model on interconnected oscillators and we discuss an application on compartmental systems. Copyright @ 2007 IFAC
\end{abstract}

Keywords: Self-organizing systems, Pattern generation, Saturation, Decentralized systems, Oscillators, Distribution networks

\section{INTRODUCTION AND MOTIVATION}

The clustering phenomenon is observed in fields ranging from the exact sciences to social and life sciences; consider e.g. swarm behavior of animals or social insects (Reynolds, 1987), opinion formation (Hegselmann and Krause, 2002) or the clusters in the frequency space for synchronized coupled oscillators (Strogatz, 2003) as a model for heart cells. We present a model that captures this phenomenon and at the same time allows a mathematical analysis. We formulate necessary and sufficient conditions for the occurrence of a given cluster structure. The model, or elaborated versions, may be used as a tool for explaining some of the phenomena mentioned above, for systematizing arguments, for prediction and for control.

We will relate the model to the Kuramoto model (Kuramoto, 1984), which describes systems of coupled oscillators. We indicate the similarities between partial entrainment of oscillators and clustering as described in our model, both for the time evolution and the dependence on the coupling strength.

We will also discuss an application on a configuration of connected basins as a specific example of a compartmental system. Compartmental systems consist of interconnected compartments, each with a certain production or consumption rate. We will consider a system of interconnected water tanks, which will almost naturally lead to the proposed model (2).

\section{THE DYNAMICS}

In general interacting agents are bound to generate unpredictable unstructured behavior. We pro- 
pose a mathematical model with a particular type of interaction such that through self-organization a structure emerges where sets of clustered agents find themselves in balance. The focus of this paper is on the dynamical behavior of this clustering phenomenon and on a characterization of the emerging cluster structure in terms of the parameters of the system.

We present a simple model admitting a succinct formulation of the conditions governing the cluster configuration at which the system settles. The differential equations for the model consisting of $N$ agents $(N>1)$ are

$$
\dot{x}_{i}(t)=b_{i}+\frac{1}{N} \sum_{j=1}^{N} f\left(x_{j}(t)-x_{i}(t)\right),
$$

$\forall t \in \mathbb{R}, \forall i \in\{1, \ldots, N\}$, with $x_{i}, b_{i} \in \mathbb{R} ; x_{i}(t)$ describes the state of agent $i$ at time $t, \dot{x}_{i}(t)$ the time derivative, and $b_{i}$ represents the autonomous component in each agent's behavior. The summation term represents the attraction exerted by the other agents on each agent. The function $f: \mathbb{R} \rightarrow \mathbb{R}$ is odd and non-decreasing with respect to distances between agents. This implies a symmetric attraction between any two agents. We assume that the interaction intensifies with separation up to a certain saturation level:

$$
\exists d>0: f(x)=F, \quad \forall x \geq d .
$$

More general saturation types will be discussed elsewhere (Aeyels and De Smet, 2007a). A Lipschitz condition on $f$ is introduced for technical reasons; it guarantees a unique solution to the differential equation with respect to a set of initial conditions.

For convenience we will present our results for the model (1), but an extension to the following generalization is possible (Aeyels and De Smet, 2007a):

$$
\dot{x}_{i}(t)=b_{i}+A_{i} \sum_{j=1}^{N} K_{i j} \gamma_{j} f\left(x_{j}(t)-x_{i}(t)\right),
$$

$\forall t \in \mathbb{R}, \forall i \in\{1, \ldots, N\}$. The function $f$ has the same characteristics as before. The interpretation of $b_{i}$ remains unaltered. The parameters $A_{i}$ and $\gamma_{i}$ are all positive. The matrix $K$ is symmetric and irreducible with $K_{i j} \geq 0$, while the diagonal elements $K_{i i}$ are all equal to zero. It is important to notice that the interaction structure of (2) is completely arbitrary, while model (1) involves all-to-all coupling. The elements of matrix $K$ represent levels of attraction between agent pairs (e.g. no attraction between agents $i$ and $j$ if $K_{i j}=$ $0)$; the extent to which each individual agent $j$ tends to attract other agents is denoted by $\gamma_{j}$. The parameter $A_{i}$ reflects the sensitivity of agent $i$ to interactions with other agents.
Although model (2) is overparametrized, we prefer to leave it unaltered since the parameters can be given physical relevance in applications.

\section{ANALYSIS AND RESULTS}

Notice that in the description of our results, we stick to model (1), but the application described later on is supported by the generalized results related to the extended model (2). Assume that, for a particular solution of (1), the behavior of the agents can be characterized as follows by an ordered set of clusters $\left(G_{1}, \ldots, G_{M}\right)$ defining a partition of $\{1, \ldots, N\}$ :

- The distances between agents in the same cluster remain bounded (i.e. $\left|x_{i}(t)-x_{j}(t)\right|$ is bounded for all $i, j \in G_{k}$, for any $k \in$ $\{1, \ldots, M\}$, for $t \geq 0)$.

- After some positive time $T$, the distances between agents in different clusters are at least $d$ and grow unbounded with time.

- The agents are ordered by their membership to a cluster: $k<l \Rightarrow x_{i}(t)<x_{j}(t), \forall i \in G_{k}$, $\forall j \in G_{l}, \forall t \geq T$.

We will refer to this behavior as clustering behavior.

For any set $G_{0} \subset\{1, \ldots, N\}$, with the number of elements denoted by $\left|G_{0}\right|$, we introduce the notation $\langle b\rangle_{G_{0}}$ for the average value of $b_{i}$ over $G_{0}$ :

$$
\langle b\rangle_{G_{0}} \triangleq \frac{1}{\left|G_{0}\right|} \sum_{i \in G_{0}} b_{i} .
$$

In (Aeyels and De Smet, 2007b) we derive the following set of necessary and sufficient conditions for clustering behavior of all solutions of the system (1), with the cluster structure $\left(G_{1}, \ldots, G_{M}\right)$ independent of the initial condition:

$$
\begin{aligned}
& \langle b\rangle_{G_{k+1}}-\langle b\rangle_{G_{k}}>\frac{F}{N}\left(\left|G_{k+1}\right|+\left|G_{k}\right|\right), \\
& \forall k \in\{1, \ldots, M-1\} ; \\
& \langle b\rangle_{G_{k, 2}}-\langle b\rangle_{G_{k, 1}} \leq \frac{F}{N}\left|G_{k}\right|, \\
& \forall G_{k, 1}, G_{k, 2} \subsetneq G_{k}, \text { with } G_{k, 2}=G_{k} \backslash G_{k, 1}, \\
& \forall k \in\{1, \ldots, M\} .
\end{aligned}
$$

The characteristics of the interaction play a key role in the proof. Since $f$ is odd all internal interactions (i.e. interactions between agents in the same cluster) cancel when calculating the velocity of the 'center of mass' of a cluster, similar to the cancellation of internal interactions in mechanics. The saturation of $f$ implies that the interactions between agents from different clusters reduce to $F / N$ or $-F / N$ whenever agents from different clusters are separated over at least a distance $d$. The monotonicity of $f$ will guarantee that the 
resulting clustering behavior is independent of the initial condition.

Under the assumption of clustering behavior and taking into account the previous considerations, the ordering of the agents and distances growing unbounded with time for agents in different clusters will lead to the condition (3). Similarly, since distances between agents from the same cluster remain bounded the condition (4) can be derived. This implies the necessity of the inequalities (3) and (4) for the existence of a solution of (1) satisfying clustering behavior. Next we give an outline of the proof of sufficiency.

The main idea is to pick an initial condition for which agents from different $G_{k}$ will always (i.e. for all $t \geq 0$ ) be separated over at least a distance $d$, with their interaction saturated as a consequence. Invoking the condition (4) it can then be shown that the differences in $x(t)$ values will be bounded for agents in the same $G_{k}$. From this boundedness together with the condition (3), it will follow that the differences in $x(t)$-values for agents from different $G_{k}$ will grow unbounded. The solution of (1) corresponding to this particular initial condition will exhibit clustering behavior (with $T=0$, and the clusters equal to the $G_{k}$ ). Any other solution $\hat{x}$ of (1) will exhibit the same clustering behavior (i.e. identical clusters, possibly a different value for $T$ ). This follows by observing that the distance in the state space $\mathbb{R}^{N}$ between $x$ and $\hat{x}$ is a non-increasing function of time: $\frac{\mathrm{d}}{\mathrm{d} t}\left(\sum_{i=1}^{N}\left(x_{i}(t)-\hat{x}_{i}(t)\right)^{2}\right) \leq 0$. We refer to (Aeyels and De Smet, 2007b) for full details.

For a solution satisfying clustering behavior with clusters $G_{1}, \ldots, G_{M}$, it is easy to verify that the average velocity $\langle\dot{x}(t)\rangle_{G_{k}}$ over cluster $G_{k}$ will be constant after some time $T$ :

$$
\langle\dot{x}(t)\rangle_{G_{k}}=\langle b\rangle_{G_{k}}+\frac{F}{N}\left(\sum_{k^{\prime}>k}\left|G_{k^{\prime}}\right|-\sum_{k^{\prime}<k}\left|G_{k^{\prime}}\right|\right),
$$

$\forall t \geq T$. Denote the right hand side by $v_{k}$. From the boundedness of the distances between agents of cluster $G_{k}$ we can derive that (3) and (4) are necessary and sufficient for every solution $x$ of (1) to satisfy:

$$
\begin{array}{r}
\exists l>0:\left|x_{i}(t)-v_{k} t\right| \leq l, \\
\forall i \in G_{k}, \forall k \in\{1, \ldots, M\}, \forall t \geq 0 .
\end{array}
$$

We also indicate that for every given set of parameters $b_{i}$ and $F$ there exists a unique ordered set partition $\left(G_{1}, \ldots, G_{M}\right)$ of clusters satisfying (3) and (4) implying a unique clustering behavior. In general there exist $N-1$ bifurcation values for the intensity of attraction $F$, defining $N$ intervals for $F$; each interval corresponds to a particular cluster configuration, and transitions to new cluster configurations take place at these bifurcation points. We refer to a forthcoming paper (Aeyels and De Smet, 2007a) in which we will extend the results in this paper to the more general system (2).

Given the model (2) and faced with the question of describing the eventually emerging cluster structure, our analysis offers two options: one can check the inequalities (3) and (4) or one can simply run a simulation of the model: the mathematical analysis guarantees convergence to a cluster structure, irrespective of the initial condition.

\section{THE KURAMOTO MODEL}

The Kuramoto model (Kuramoto, 1984) was introduced to describe synchronization in systems of coupled oscillators. We refer to (Strogatz, 2003) for its relation to flashing fireflies, pacemaker cells, Josephson junctions. The system equations are

$$
\dot{\theta}_{i}(t)=\omega_{i}+\frac{K}{N} \sum_{j=1}^{N} \sin \left(\theta_{j}(t)-\theta_{i}(t)\right),
$$

$\forall i \in\{1, \ldots, N\}, \forall t \in \mathbb{R}$. The natural frequencies $\omega_{i}$ are drawn randomly from a distribution $g$. Kuramoto showed that, for $N \rightarrow \infty$, and with $g$ unimodal and even about a value $\Omega$, there is a critical value for the coupling strength $K$ above which a solution exists exhibiting partial synchronization. For this solution a group of oscillators is moving at the same frequency $\Omega$, while the remaining oscillators are moving with different (average) frequencies. Details can be found in (Strogatz, 2000).

For finite $N$, simulations indicate the following. For a fixed $K>0$ the oscillator population can be partitioned into different subsets of which the members have bounded phase differences and have the same long term average frequency: the system exhibits partial entrainment (Aeyels et al., 2005). The partition and the associated average frequencies are independent of the initial condition for most choices of the natural frequencies and the coupling strength. When the coupling strength is increased the average frequencies of the different entrained subsets move towards each other, and when a critical value for $K$ is passed and two characteristic frequencies coincide, the corresponding entrained subsets merge into a new entrained subset. This scenario is repeated until there is full entrainment for $K$ sufficiently large, i.e. all phase differences are bounded. For more information on the stability properties of this latter solution, see (Aeyels and Rogge, 2004).

This behavior is very similar to that of the model (1), where the clusters are also independent of the initial condition and the transitions between the different clusters for varying $F$ are similar. For 


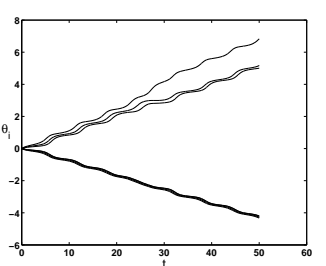

(a)

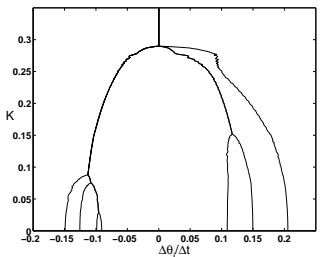

(c)

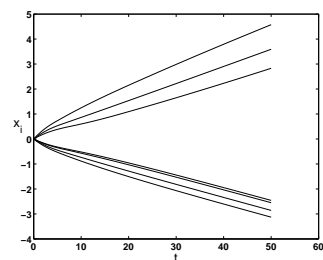

(b)

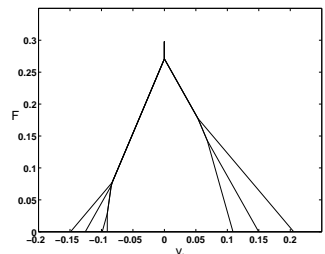

(d)
Fig. 1. Comparison of the Kuramoto model (left column) with the model (1) (right column): The first row shows the time evolution of the oscillators/agents. The second row shows the evolution of the average frequencies/velocities for varying coupling strength. The parameters are: $b=\omega=(-0.149$, $-0.126,-0.099,-0.091,0.109,0.150,0.206)$, $K=0.2$ (Fig. (a)) and $F=0.15$ (Fig. (b)).

comparison Fig. 1 shows the time evolution for a particular configuration for the Kuramoto model (Fig. 1(a)) and the analogue for the model (1) (Fig. 1(b)), as well as the evolution of the characteristic frequencies/velocities for both models in terms of the coupling strength (Fig. 1(c) and $1(\mathrm{~d})$ ). There is a remarkable (qualitative) correspondence between both models.

\section{COMPARTMENTAL SYSTEMS: INTERCONNECTED WATER BASINS}

We consider $N$ different basins connected by horizontal pipes; each basin is subject to a constant external inflow or outflow of water. We assume that the pipes have a maximal throughput, which is independent of the direction of the flow, and denoted by $K_{i j}$ for the pipe connecting basin $i$ and $j$. Representing the water height of basin $i$ by $x_{i}$, we model the volume flow rate through the connecting pipe by $K_{i j} f\left(x_{j}-x_{i}\right)$, with the saturation value $F$ is equal to 1 . (For simplicity we assume that $f$ is independent of $i$ and $j$, but the results can be extended to the general case.) The absence of a pipe between basins $i$ and $j$ corresponds to $K_{i j}=0$.

With the inflow of basin $i$ denoted by $Q_{i}$ and its surface area - which we assume to be water level independent — by $S_{i}$, one derives

$$
\dot{x}_{i}(t)=\frac{Q_{i}}{S_{i}}+\frac{1}{S_{i}} \sum_{j=1}^{N} K_{i j} f\left(x_{j}(t)-x_{i}(t)\right),
$$

$\forall i \in\{1, \ldots, N\}, \forall t \in \mathbb{R}$, which is the model (2) with $b_{i}=\frac{Q_{i}}{S_{i}}, \gamma_{i}=1$, and $A_{i}=\frac{1}{S_{i}}$ for all $i$. The velocity $v_{k}$ associated with cluster $G_{k}$ can be calculated as

$$
\begin{gathered}
v_{k}=\frac{1}{\sum_{i \in G_{k}} S_{i}} \sum_{i \in G_{k}}\left(Q_{i}+\sum_{k^{\prime}>k} \sum_{j \in G_{k^{\prime}}} K_{i j}\right. \\
\left.-\sum_{k^{\prime}<k} \sum_{j \in G_{k^{\prime}}} K_{i j}\right) .
\end{gathered}
$$

We are interested in checking whether a network of connected basins is prone to flooding. We assume that the total external inflow equals the total external outflow, and therefore the desired behavior corresponds to one cluster $G_{1}=\{1, \ldots, N\}$ with velocity $v_{1}=\frac{1}{\sum_{i \in G_{1}} S_{i}} \sum_{i \in G_{1}} Q_{i}=0$. For any solution with more than one cluster, one of the generalizations of the inequalities (4) to the extended model (2) (see (Aeyels and De Smet, 2007a)) will be violated, expressing that there exists a partition of the set of basins into two non-empty subsets, for which the production in one subset cannot be transported to the other subset by the interconnections between them. Some of the basins will overflow; these basins are associated to the clusters with a positive $v_{k}$-value.

As an illustration, we consider a configuration of $N=7$ basins all having the same surface area, equal to 1 , implying that $A_{i}=1$, and furthermore that $b_{i}$ equals the external inflow rate of basin $i$. (For simplicity we will omit units.) The vector $b$ containing the $b_{i}$-values and the matrix $K$ containing the $K_{i j}$-values are given by

$$
b=\left[\begin{array}{c}
-4 \\
0 \\
-1 \\
-1 \\
1 \\
2 \\
3
\end{array}\right], \quad K=\left[\begin{array}{lllllll}
0 & 1 & 0 & 1 & 0 & 1 & 0 \\
1 & 0 & 1 & 0 & 0 & 0 & 0 \\
0 & 1 & 0 & 0 & 0 & 0 & 1 \\
1 & 0 & 0 & 0 & 1 & 0 & 1 \\
0 & 0 & 0 & 1 & 0 & 1 & 0 \\
1 & 0 & 0 & 0 & 1 & 0 & 0 \\
0 & 0 & 1 & 1 & 0 & 0 & 0
\end{array}\right] .
$$

A simulation (Fig. 2(a)) reveals that this interconnection structure is not able to prevent basins from overflowing. The objective might be to alter the connection structure by adding a minimal number of pipes (of maximal throughput 1) in order to avoid flooding, i.e. in order to obtain a single cluster at zero velocity.

Figure 2(a) shows that there are 5 different clusters: $G_{1}=\{1\}, G_{2}=\{2,3\}, G_{3}=\{4\}, G_{4}=$ $\{5,6\}, G_{5}=\{7\}$. Adding an extra pipe will not affect the velocity of the cluster with largest (resp. smallest) $v_{k}$-value unless the pipe is connected to one of the basins in this cluster. Therefore if one extra connection would be sufficient it would have to connect basins 1 and 7. Simulation with this extra connection results in 3 clusters: $G_{1}^{\prime}=\{1,2,3\}$, $G_{2}^{\prime}=\{4,7\}, G_{3}^{\prime}=\{5,6\}$ (see Fig. 2(b)), implying 


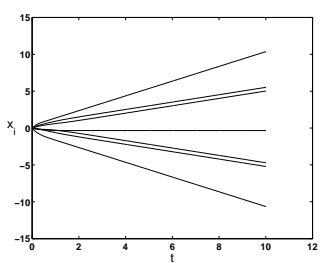

(a)

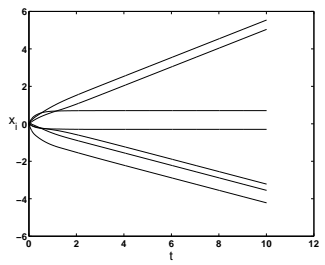

(b)
Fig. 2. Compartmental systems: Fig. (a) shows the time evolution of the $x_{i}$ resulting from the initial configuration. Fig. (b) shows the time evolution of the $x_{i}$ when an extra pipe is added between basins 1 and 7 .

that we still need (at least) one extra connection between a basin from $G_{1}^{\prime}$ and a basin from $G_{3}^{\prime}$. Simulations show that any extra connection between either 1, 2 or 3 and 5 or 6 lead to one cluster at zero velocity, solving the problem.

This example shows that the model (2) can be helpful for analyzing compartmental systems and systematizing the search for solutions to associated problems.

\section{ACKNOWLEDGMENTS}

This paper presents research results of the Belgian Programme on Interuniversity Attraction Poles, initiated by the Belgian Federal Science Policy Office. The scientific responsibility rests with its authors.

Filip De Smet is a Research Assistant of the Research Foundation - Flanders (FWO - Vlaanderen).

\section{REFERENCES}

Aeyels, Dirk and Filip De Smet (2007a). Clustering in a network of non-identical and attractive agents. In preparation.

Aeyels, Dirk and Filip De Smet (2007b). A mathematical model for the dynamics of clustering. Submitted to SIAM Review.

Aeyels, Dirk and Jonathan A. Rogge (2004). Existence of partial entrainment and stability of phase locking behavior of coupled oscillators. Progress of Theoretical Physics 112(6), 921942.

Aeyels, Dirk, Filip De Smet and Bavo Langerock (2005). Partial entrainment of oscillators in the finite Kuramoto model. In: Proceedings of 2005 International Symposium on Nonlinear Theory and its Applications. Research Society of Nonlinear Theory and its Applications, IEICE.

Hegselmann, Rainer and Ulrich Krause (2002). Opinion dynamics and bounded confidence: models, analysis and simulation. Journal of Artificial Societies and Social Simulation.

Kuramoto, Y. (1984). Cooperative dynamics of oscillator community. Prog. Theoret. Phys. Suppl. 79, 223-240.

Plapp, John E. (1968). Engineering Fluid Mechanics. Series in Engineering of the Physical Sciences. Prentice Hall.

Reynolds, Craig W. (1987). Flocks, herds, and schools: A distributed behavioral model. Computer Graphics 21(4), 25-34. (SIGGRAPH '87 Conference Proceedings).

Strogatz, Steven H. (2000). From Kuramoto to Crawford: exploring the onset of synchronization in populations of coupled oscillators. Physica D 143, 1-20.

Strogatz, Steven H. (2003). SYNC: The Emerging Science of Spontaneous Order. Hyperion Press. 